\title{
Metastasis of choroidal melanoma to the contralateral choroid, orbit, and eyelid*
}

\author{
JERRY A SHIELDS,' CAROL L SHIELDS,' ERIC P SHAKIN,' \\ AND LARRY E KOBETZ \\ From the 'Ocular Oncology Service, Wills Eye Hospital, Jefferson Medical College, Thomas Jefferson \\ University, Philadelphia, USA, and ${ }^{2}$ St Joseph's Hospital, Hamilton, Ontario, Canada
}

SUMMARY A 52-year-old woman was found to have a small juxtapapillary pigmented lesion in the choroid of the left eye. This lesion remained clinically stationary for one year, but subsequent growth prompted enucleation of the eye. The tumour was diagnosed histologically as a choroidal malignant melanoma of mixed cell type. Approximately 52 months later the patient developed proptosis of the contralateral (right) eye. Orbital ultrasonography showed a large mass in the right orbit, which was confirmed by needle biopsy to be a melanoma. In addition the patient was found to have metastatic melanoma to the choroid, right lower eyelid area, and liver. The development of simultaneous orbital, choroidal, and eyelid metastases from a contralateral choroidal melanoma is of ophthalmic interest and appears to be unique.

Metastatic disease from primary uveal malignant melanoma characteristically occurs in the liver and less commonly in the lung, subcutaneous tissues, and other organs.' Metastatic uveal melanoma to the opposite eye is extremely rare. We report a unique case of metastasis of a relatively small choroidal melanoma from the choroid of the left eye to the contralateral orbit, choroid, and eyelid.

\section{Case report}

A 52-year-old white woman had no prior ocular problems until April 1979 when she was found on routine examination to have a small juxtapapillary pigmented choroidal mass in the left eye. In October 1978 a fundus examination of both eyes by indirect ophthalmoscopy had shown no abnormalities. On our examination in April 1979 her visual acuity was 6/6 $(20 / 20)$ in each eye, and the intraocular pressures were normal. The fundus of the right eye was carefully examined by indirect ophthalmoscopy, and there was no evidence of choroidal naevi or other fundus lesions.

In the left eye a moderately pigmented choroidal tumour extended superiorly from the optic disc

${ }^{*}$ Presented at the Annual Meeting of the Atlantic Coast Fluorescein Club, Baltimore, 7 February 1986.

Correspondence to Dr J Shields, Oncology Service, Wills Eye Hospital, Ninth and Walnut Streets, Philadelphia, Pa 19107, USA. margin; it measured approximately $6 \times 5 \mathrm{~mm}$ in basal diameter and $2.5 \mathrm{~mm}$ in thickness (Fig. 1). There was a geographical distribution of orange pigment over the surface of the tumour. Fluorescein angiography

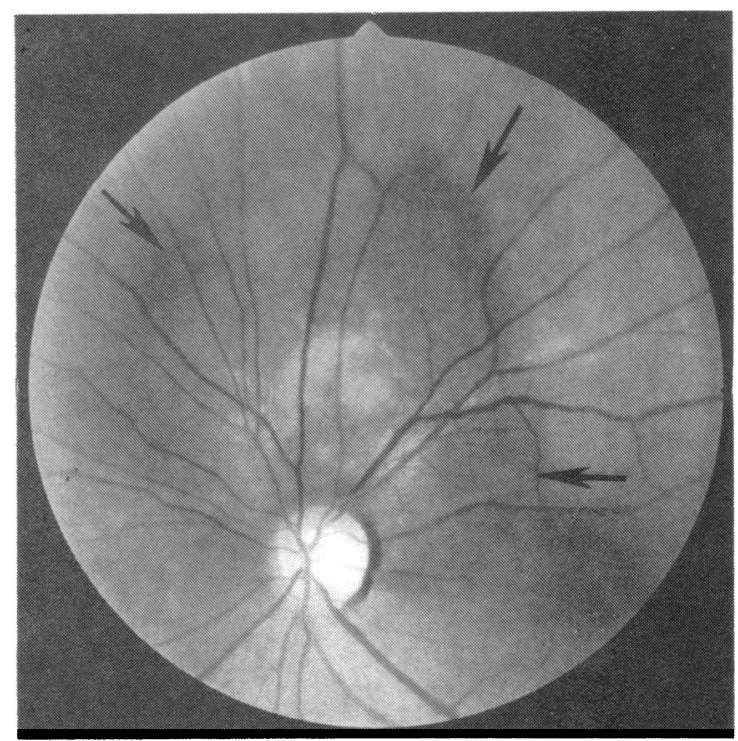

Fig. 1 Clinical photograph of small peripapillary pigmented lesion (arrows) as seen on the initial visit. 


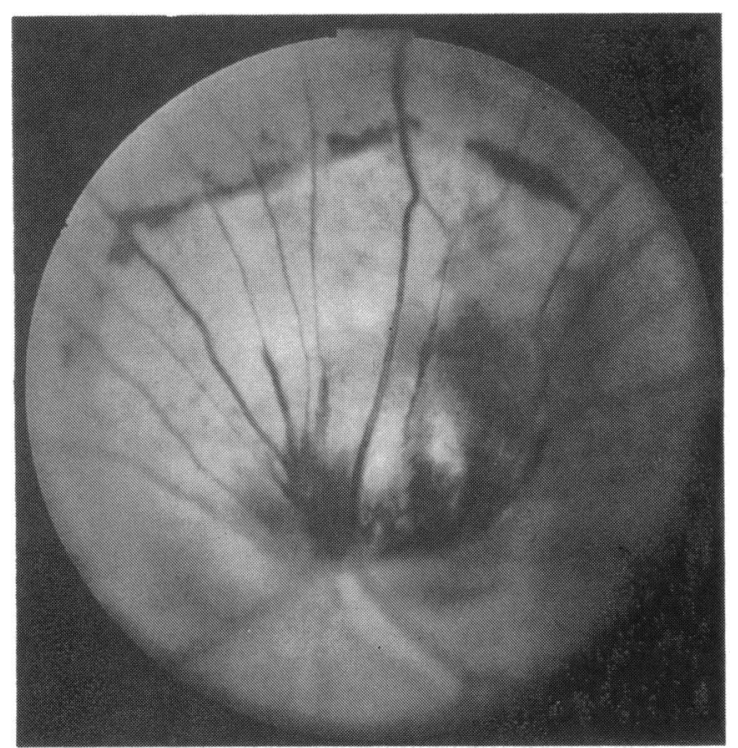

Fig. 2 Fundus photograph of same lesion as shown in Fig. 1 after progressive growth. Note that the lesion is now obscuring the superior margin of the optic disc.

showed early mottled fluorescence and late staining of the mass compatible with a suspicious naevus or small melanoma. Ultrasonography demonstrated a solid choroidal tumour with low internal reflectivity and a thickness of $2.5 \mathrm{~mm}$.

These findings were compatible with a suspicious choroidal naevus or a small choroidal melanoma and the patient was followed up periodically. Examina-

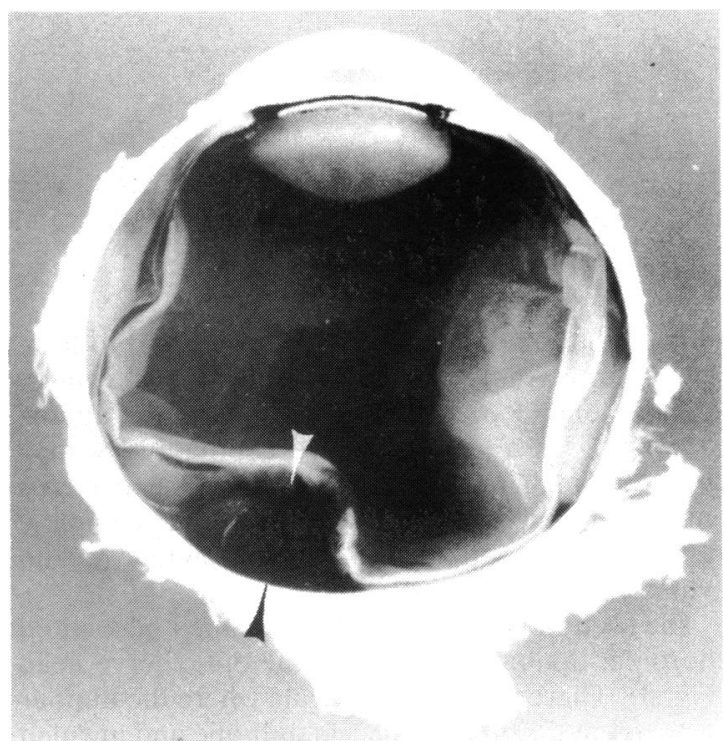

Fig. 3 Grossly sectioned eye showing pigmented tumour in the posterior segment (arrows).

tions were performed every 4-6 months and the lesion remained unchanged. Ocular and general physical examinations revealed no other abnormalities.

On 14 October 1980 her visual acuity remained 6/6 in each eye but the tumour had become more elevated and extended over the superior margin of the optic disc (Fig. 2). Repeat ultrasonography

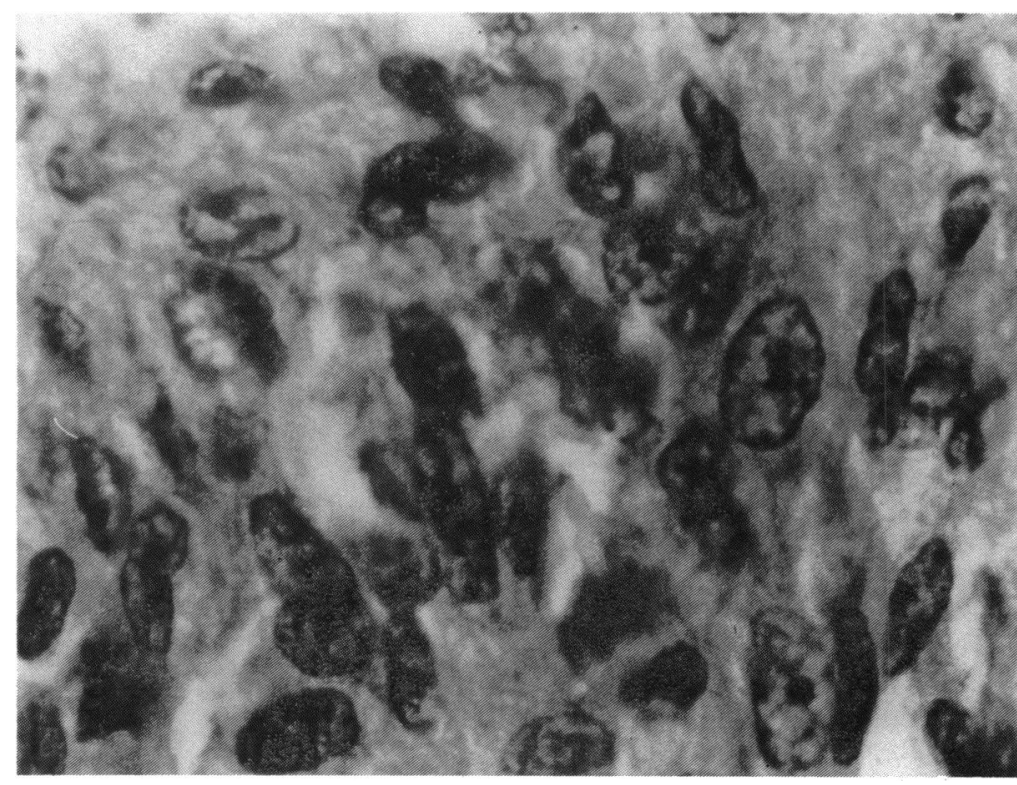

Fig. 4 Histopathology of choroidal tumour showing predominance of spindle melanoma cells. Haematoxylineosin, $\times 250$. 


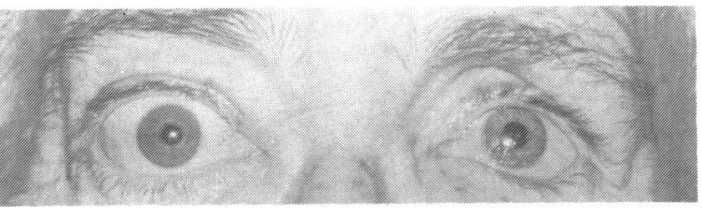

Fig. 5 Clinical appearance of patient showing ocular prosthesis on the left and proptosis on the right side.

showed the tumour to be $5.4 \mathrm{~mm}$ thick and to have low internal reflectivity and acoustic hollowness. An incisional P-32 test revealed a positive uptake of $175 \%$. These findings were compatible with a choroidal malignant melanoma, and the eye was enucleated on the following day. A complete systemic evaluation prior to enucleation revealed no evidence of metastatic disease.

The sectioned eye had a dome shaped pigmented choroidal tumour arising near the optic nerve head (Fig. 3). There was a small nodule where the tumour had broken through Bruch's membrane and invaded the sensory retina. Microscopically the tumour proved to be a mixed-cell malignant melanoma with a predominance of spindle B cells (Fig. 4).

Follow-up examination in April 1981, October 1981, April 1982, and May 1983 showed the patient to be systemically normal with no evidence of metastatic disease or local recurrence. On 27 October 1983 her liver enzymes were found to be slightly raised, with a lactate dehydrogenase level of $249 \mathrm{IU} / \mathrm{l}$

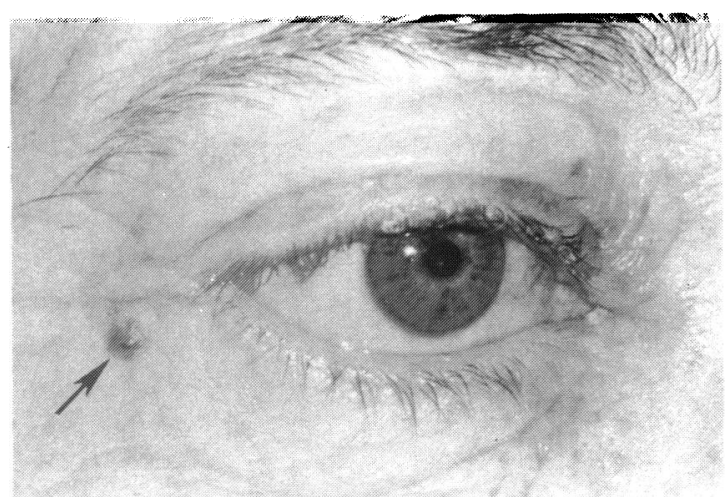

Fig. 7 Clinical appearance of metastatic melanoma to lateral aspect of right lower eyelid (arrow).

(normal 100-225), a serum glutamic oxaloacetic transaminase level of $88 \mathrm{IU} / 1$ (normal 7-40) and a $\gamma$ glutamyl transpeptidase level of 24 IU/l (normal 6-24). A systemic examination was recommended at that time, but the patient did not return for follow-up until 22 May 1984, at which time she complained of progressive epigastric pain and weight loss. She was noted to have tenderness of the right upper abdominal quadrant and pigmented lesions on the back and scalp. One of these was biopsied and found to be metastatic melanoma. A liver scan revealed multiple abnormal defects compatible with metastatic melanoma.

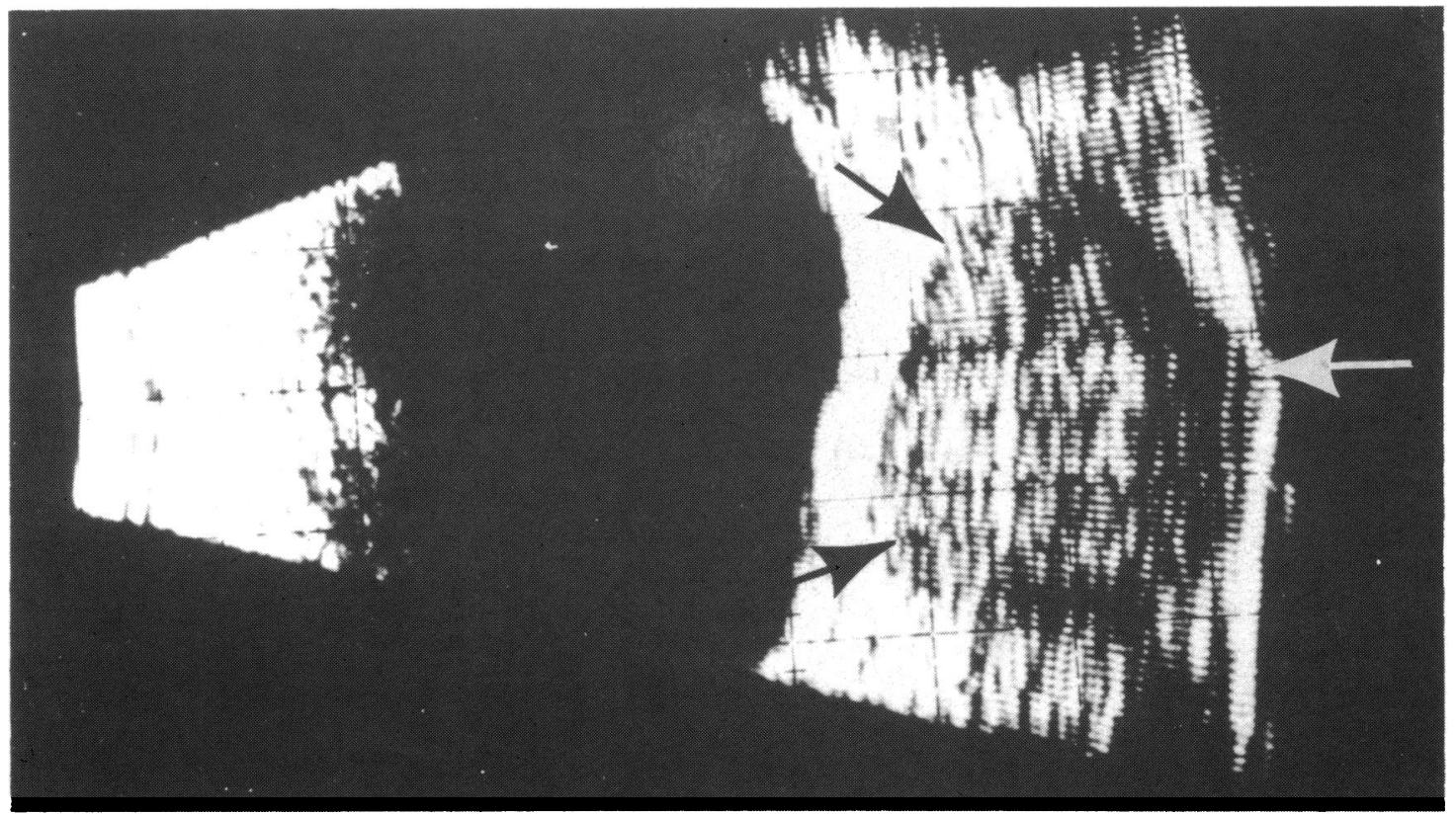

Fig. 6 B scan ultrasonogram showing rounded retrobulbar mass (arrows) with moderately high internal reflectivity. 


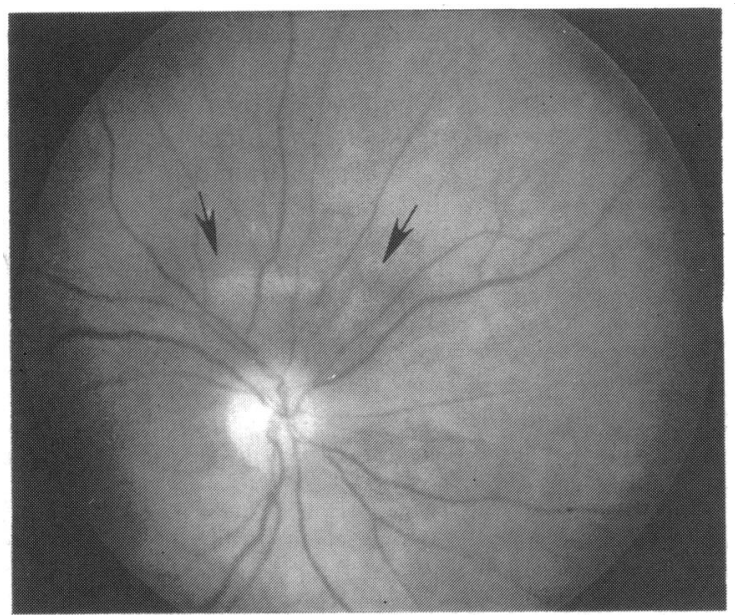

Fig. 8A Fundus photograph showing metastatic choroidal melanoma superior to optic disc in the right eye (arrows).

The patient was treated by systemic chemotherapy and remained fairly stable until 18 April 1985, when she first noted prominence of her right eye. Examination disclosed $8 \mathrm{~mm}$ of proptosis (Fig. 5) of right eye by Hertel exophthalmometry. B scan ultrasonography showed a rounded retrobulbar mass with high internal reflectivity (Fig. 6). There was also a new black subcutaneous nodule in the lateral aspect of the right lower eyelid (Fig. 7). Fundus examination revealed prominent choroidal folds and two pigmented choroidal tumours, one superonasal to the optic disc and the other inferotemporal to the fovea (Figs. 8A, 8B). An orbital fine needle aspiration biopsy of the retrobulbar mass showed metastatic melanoma (Fig. 9).

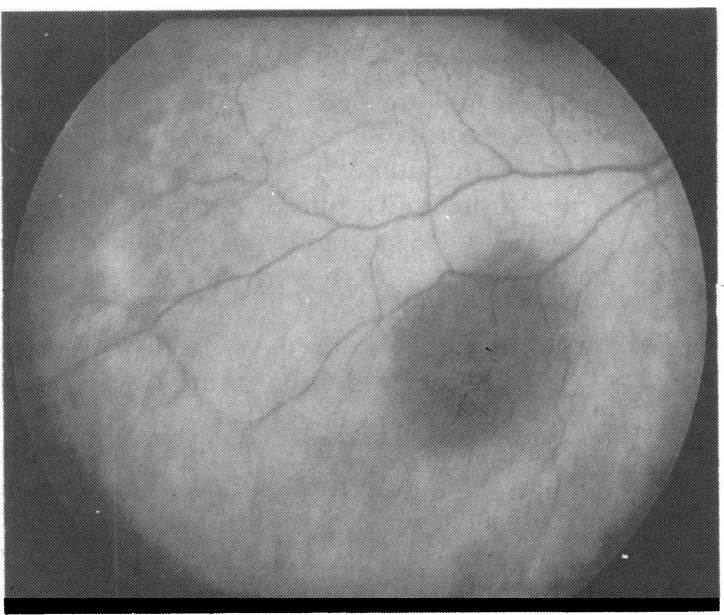

Fig. 8B Fundus photograph showing metastatic choroidal melanoma inferotemporal to macular area in the right eye.

It was recommended that the patient have continued chemotherapy and radiation to the right eye and orbit. However, this was never completed, and she died with liver failure on 16 May 1985. Permission for necropsy was not granted.

\section{Discussion}

With the exception of orbital extension of uveal melanomas, malignant melanomas of the orbit are extremely rare. Melanomas can secondarily extend directly into the orbit from the primary lesions in the uvea, conjunctiva, and eyelid..$^{1-3}$ Metastatic cutaneous melanoma to the orbit is quite rare. In the series of 764 orbital tumours reported by Henderson

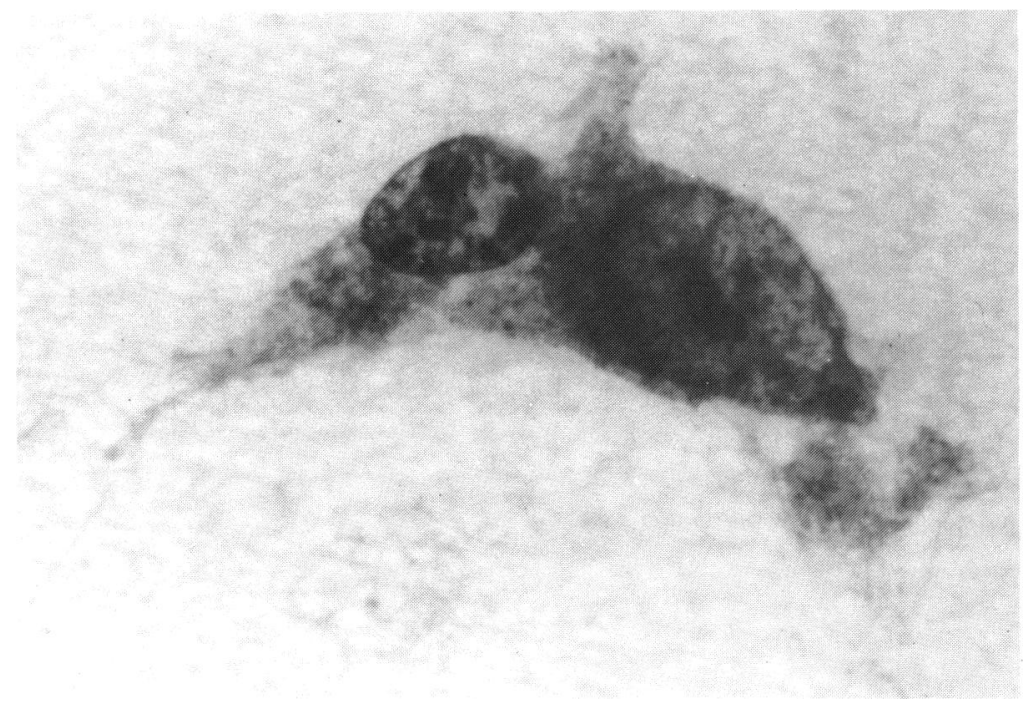

Fig. 9 Cytologyof fine needle aspiration biopsy showing anaplastic tumour cells with cytoplasmic melanin consistent with malignant melanoma cells. Papanicolaou, $\times 320$. 
and Farrow there was one case of metastatic cutaneous melanoma to the orbit and no cases of metastatic uveal melanoma to the orbit. ${ }^{4}$ In 1967 Font and associates recorded five cases of orbital involvement by metastatic cutaneous melanoma in the files of the Armed Forces Institute of Pathology. ${ }^{5}$ In the series of 645 biopsied orbital masses reported by Shields and associates there were no cases of metastatic melanoma to the orbit. ${ }^{6}$

To our knowledge there have been only two reported cases in the English literature of choroidal melanoma metastatic to the opposite orbit. ${ }^{78}$ In the case reported by Philps in 1949 an orbital metastasis developed approximately 10 years after enucleation of the opposite eye for a large choroidal melanoma. In the case reported by Foster and associates in 1957 metastasis to the opposite orbit was noted approximately four years after enucleation of a uveal melanoma. $^{8}$

Sceptics might argue that the eyelid, orbital, and choroidal melanomas in this case could represent metastases from either an undetected cutaneous melanoma or from an occult visceral melanoma. Metastatic cutaneous melanoma to the choroid, although quite rare, is well recognised. ${ }^{59}$ Metastatic melanoma to the orbit has been known to occur from an occult primary melanoma in the colon. ${ }^{10}$ However, our patient had thorough general examinations, and there was no evidence of a primary cutaneous or visceral melanoma.

We are not aware of any prior well documented cases of metastatic uveal melanoma to the contralateral choroid. The case reported here appears to represent a well documented example of metastases from a relatively small but growing melanoma of the choroid to the contralateral orbit, choroid, and eyelid, as well as systemic metastases. It is possible that other patients with near terminal melanomatosis from uveal melanomas have metastatic foci to the opposite choroid or orbit, but that they are not subject to detailed ocular examination, and the metastatic foci remain subclinical.

This work was supported in part by the Ocular Oncology Fund, Wills Eye Hospital, in part by the Oncology Research Fund, Wills Eye Hospital, and in part by the Black Patch Invitational Golf Tournament, Downingtown, Pa, USA.

\section{References}

1 Shields JA. Diagnosis and management of intraocular tumors. St Louis: Mosby, 1983: chapter 7.

2 Shields JA, Augsburger JA, Corwin S, Donoso LA, DiSantis M. The management of uveal melanomas with extrascleral extension. Orbit 1986; 5: 31-7.

3 Shields JA. Malignant melanomas involving the orbit. In: Hornblass A, ed. Ophthalmic and orbital plastic and reconstructive surgery. Baltimore: Williams and Wilkins, in press.

4 Henderson JW, Farrow GM. Orbital tumors. New York: Decker, 1980.

5 Font RL, Naumann G, Zimmerman LE. Primary malignant melanoma of the skin metastatic to the eye and orbit: report of ten cases and review of the literature. Am J Ophthalmol 1967; 63: 738-54.

6 Shields JA, Bakewell B, Augsburger JJ, Flanagan JC. Classification and incidence of space-occupying lesions of the orbit. A survey of 645 biopsies. Arch Ophthalmol 1984; 102: 1606-11.

7 Philps S. Choroidal sarcoma with metastasis in the opposite orbit. Br J Ophthalmol 1949; 33: 732-9.

8 Foster J, Henderson W. Cowie JW, Harriman DSF. Choroidal sarcoma with metastasis in the opposite orbit. Br J Ophthalmol 1957; 41: 42-7.

9 DeBustros S, Augsburger JJ, Shields JA, Shakin EP, Pryor C. Intraocular metastases from cutaneous malignant melanoma. Arch Ophthalmol 1985; 103: 937-40.

10 Prasad VN, Bist HK, Narain M. Metastatic orbital malignant melanoma-a cause of unilateral proptosis. Afro-Asian $J$ Ophthalmol 1984; 3: 87-9.

Accepted for publication 15 April 1987. 